\title{
Prothèses implantables: faut-il prévenir ? oui mais qui ?
}

\author{
Implantable prostheses: should we prevent? Yes but who?
}

\author{
D. Heresbach \\ C) Springer-Verlag France 2012
}

Suite aux problèmes soulevés par les prothèses mammaires de type PIP, nous avons tous eu une pensée pour les prothèses digestives que nous implantons. Sans aucun parallèle puisque les éléments actuels laissent à penser qu'il ne s'agit pas d'une usure prématurée ni d'une utilisation déviante d'un dispositif médical (DM) mais plutôt d'un « défaut de fabrication », cette affaire nous interpelle surtout sur les mesures ou réactions qui seront mises en place pour éviter la récidive d'un tel « événement ». Il faut rappeler que les DM classés en quatre groupes comportent la classe I (seringues, cannes, fauteuil roulant), IIa (lentilles de contact, prothèses auditives, sonde urinaire), IIb (fils pour suture non résorbable, scanner, pompe à insuline externe) et III (prothèse de hanche ou genoux, stent coronaire, valves cardiaques, défibrillateur ou PM implantable, stérilet, implant mammaire, DM implantable actif). Les prothèses digestives classées en DM IIb ont des indications variées qui, quel que soit l'étage digestif, se décomposent en indications temporaires pour sténose, voire perforation bénigne et indications définitives pour sténose, voire perforation maligne. Le paradoxe veut que les indications définitives sont, étant donné le pronostic ou comorbidités, palliatives et donc temporaires. Dans le cas des prothèses œesphagiennes qui représentent l'indication la plus importante et usuelle, si la multitude des DM a été source de progrès pour tous ceux qui ont connu la dilatation à la bougie avant pose de prothèse de «Celestin » en cas de cancer $\mathrm{T} 3$ ou souvent $\mathrm{T} 4$, disposons-nous à ce jour d'éléments objectifs (au minimum), voire scientifiques (idéal) ou validés par l'Afssaps (mieux ou pire pour certains) ? Si nous disposons depuis 1998 de dix métaanalyses ou « recommandations » et, entre 1993 et 2011, parmi 165 études cliniques, de 63 essais contrôlés randomisés, les études contrôlées et randomisées ne comparent pas des prothèses identiques de fabricants différents mais plutôt une prothèse avec une potentielle amélioration à la référence précédente du même fabricant ! Alors que sous la pression

\section{Heresbach $(\bowtie)$}

Centre hospitalier de Cannes, unité d'endoscopie digestive, 15 avenue des Broussailles, CS 50008, F-06414 Cannes e-mail : d.heresbach@ch-cannes.fr des « utilisateurs ou de leurs associations », les bénéficiaires des prothèses mammaires arguent qu'ils n'ont pas choisi la marque ou le fabricant de leur prothèse, pouvons-nous imaginer, annoncer à un patient : « j'ai deux nouvelles, la mauvaise c'est que vous avez un cancer de l'œsophage que l'on ne peut pas opérer, et la bonne c'est que vous pouvez choisir la marque et le fabricant de votre tuyau métallique appelé prothèse, dont la matério-vigilance a décrit $\mathrm{X} \%$ de rupture dans l'aorte et $\mathrm{Y} \%$ de perforation dans les bronches ou la trachée »!

Espérons que le tout sécuritaire ne nous amènera pas à cet extrême ou, par populisme, introduira la notion selon laquelle le poseur de prothèse est responsable du choix et donc de la qualité de la prothèse délivrée ; cette hypothèse demeure malheureusement la plus probable. Dans ce dernier cas, la responsabilité sera-t-elle partagée par le pharmacien d'établissement qui, actuellement en accord avec les poseurs de prothèse, choisissent le DM et son fabricant, souvent dans le cadre d'un MAPA, qui permet l'équilibre du poste budgétaire des DM. Inversement, la solution serait-elle de demander au patient d'aller en pharmacie de ville, choisir et acheter sa prothèse, procédure peu en vogue en France dans le cadre d'une déresponsabilisation du patient ? La SFED doit-elle attendre que le sujet soit moins chaud ou doit elle prévenir » que :

- il n'existe pas, à « design et taille » identiques, d'arguments ou d'études comparatives de fabricants différents?;

- la rareté des événements indésirables graves rend illusoire tout essai comparatif apportant une preuve EBM ;

- les prothèses digestives sont implantables mais temporaires, y compris dans les indications dites définitives et à ce titre, doivent demeurer en classe IIa ou IIb, bien qu'intracoporelles mais « non implantables ».

Les premières réactions sur le sujet relatent une homogénéisation de la réglementation européenne prévue en 2012. Celle-ci, outre une réglementation et un contrôle plus stricts des organismes conférant le marquage $\mathrm{CE}$, propose une phase d'observation après délivrance du marquage CE pour réaliser une sorte de phase IV appliqué aux DM. On ne peut 
que soutenir cette hypothèse déjà mise en œuvre par la SFED par le biais de registres ou observatoires : mucosectomie digestive, prothèse œsophagienne plastique temporaire, dissection sous-muqueuse, radiofréquence œsophagienne, microscopie confocale, vidéocapsule colique. Néanmoins, ces observatoires sont souvent possibles grâce au concours du fabricant ou distributeur du DM qui, autant qu'une phase IV, soutient ces actions afin de diffuser la méthode au prix d'une mise à disposition gracieuse ou à prix coutant du DM. Dans la stratégie institutionnelle, on est bien loin de la question centrale à tout nouveau DM ; son financement. On ne connaît que trop bien le duo HAS-CPAM qui pour la première octroyait, après saisine par une société savante, un code sans cote et la seconde ne donnant une cote ou valorisation qu'après démonstration d'un service médical rendu supérieur à la méthode ou DM en place. Si cette dernière attitude ne peut être qu'approuvée, il existe un « espace mort » entre le marquage CE (début de la phase IV) et le remboursement du DM (après la fin de la phase IV si sa conclusion confirme le "bénéfice médical rendu ») qui est lié à la valorisation de l'acte. Nous savons très bien que même les budgets pour la recherche clinique ne sont pas en mesure de couvrir le coût des DM pour cette phase IV malgré tout l'affichage politique dont ils font l'objet. Alors, oui il faut prévenir, mais qui ?
- L'afssaps ou les politiques en charge du dossier européen, mais est-ce vraiment leur préoccupation à l'heure des chaises musicales?

- Les patients pour leur préciser et avertir de l'absence de critères objectifs pouvant soutenir un choix ou une comparaison, mais est-ce vraiment leur préoccupation à l'heure des déremboursements de frais de santé?

- Le futur président ou ministre de la Santé, mais est-ce vraiment leur préoccupation, sauf si on anticipe que la perte d'innovation ou d'évaluation des DM serait source de délocalisation des soins?

Conflit d'intérêt : le Pr D. Heresbach a réalisé des missions ponctuelles d'expert auprès des laboratoires Norgine, Aptalis en 2010 et de la société MedPass, des laboratoires Norgine et Aptalis en 2011. Il a participé comme rédacteur en chef à un symposium organisé par Mauna Kea Technology en 2010 et Ella SA en 2011 et participera au CFE de 2012 avec le soutien de Wilson-Cook France. Il est coordonnateur du registre des polypes d'Ille-et-Vilaine (2009-2010) en partenariat à hauteur de $30 \%$ avec les laboratoires Ferring SA et d'un STIC financé par l'INCa en 2009-2010 sur la coloscopie virtuelle. 